Research Article

\title{
Statistical Model of Normal Contact Stiffness of Fixed Joint Surface during Unloading after First Loading
}

\author{
Donghua Yin, Xueliang Zhang ${ }^{D}$, Yonghui Chen, Guosheng Lan, Yanhui Wang, \\ and Shuhua Wen \\ School of Mechanical Engineering, Taiyuan University of Science and Technology, Taiyuan 030024, China \\ Correspondence should be addressed to Xueliang Zhang; 451695404@qq.com
}

Received 21 October 2020; Revised 7 February 2021; Accepted 13 February 2021; Published 29 April 2021

Academic Editor: Jun Liu

Copyright $\odot 2021$ Donghua Yin et al. This is an open access article distributed under the Creative Commons Attribution License, which permits unrestricted use, distribution, and reproduction in any medium, provided the original work is properly cited.

\begin{abstract}
Based on Kogut and Etsion's model (KE model), a statistical method is used to establish a model of normal contact stiffness of fixed joint surface during unloading after first loading. Simulation results show that, for the elastoplastic contact, normal contact stiffness of joint surface is the nonlinear function of mean surface separation during loading and unloading and decreases as the separation increases. For different plasticity indexes, the normal contact stiffness of joint surface varies differently following the change of mean surface separation during loading and unloading.
\end{abstract}

\section{Introduction}

The machine tool is the mother of manufacturing industry, which is assembled by various components. There are some parts that contact each other, namely joint surface. Among them, the fixed joint surface is one of the widely existing joint surfaces. Research showed that [1] the ratio of contact stiffness of fixed joint surface to total stiffness of the whole machine was more than $60 \%$. Therefore, it is of great significance to establish a more accurate contact stiffness model for the analysis of static and dynamic characteristics of the machine tool structure.

At present, many scholars at home and abroad have studied the loading and unloading model of fixed joint surfaces. In 2002, Lin and Yui [2] studied frictionless contact between rigid sphere and elastic-fully plastic half-space or elastic-fully plastic sphere and rigid plane, and proposed loading and unloading models. In 2003, Yan and Li [3] used a nonlinear finite element method to conduct numerical research and analysis on the process of periodic indentation by a rigid sphere in the elastic-fully plastic half-space. In 2004, Jones [4] proposed two basic models for loading and unloading of an elastic-plastic rough surface. Unfortunately, none of the above important research work gave the general solution of the elastoplastic sphere during unloading. In view of this, in 2005, Etsion et al. [5] studied the unloading of loading contact between an elastic-plastic sphere and a rigid plane loading using a finite element method and gave a dimensionless relationship between unloading load and deformation of the sphere, as well as dimensionless expressions of residual interference and residual curvature of the sphere after fully unloading, which has universal theoretical guiding significance. In 2006, based on the research work by Etsion et al. [5], Kadin et al. [6] further proposed an unloading contact statistical model of rough surface elasticplastic contact under a single loading and unloading cycle. In 2010, based on the research work by Etsion et al. [5], Zait et al. [7] presented empirical expressions of residual interference, contact area, and contact load of the sphere considering unloading of elastoplastic sphere affected by adhesive contact. In 2012, Zhao et al. [8] studied the frictionless contact between a power-law hardening elastoplastic sphere and a rigid plane and gave the relationship between contact load and deformation of the sphere, as well as the expression of residual interference and other variables during unloading. However, all the above research studies were aimed at loading and unloading contact models of sphere and plane. In 2017, Fu et al. [9] established a static loading and unloading contact model based on the elasticplastic unloading contact model of the rough surface of the 
KE model. In 2017, An et al. [10] established a statistical model of normal contact stiffness of joint surface during loading and unloading using statistical methods. Because deformation mechanism has an important influence on the normal contact stiffness of fixed joint surface in the elasticplastic contact problem, the accuracy degree of the model obtained by fitting the finite element analysis curve of each stage of elastic-plastic contact evolution has a great influence on the normal contact stiffness. However, Fu et al. [9] and An et al. [10] only used an empirical expression to describe the deformation in the elastic-plastic stage, so its accuracy needs to be improved. Recently, Nassiraei et al. [11-13] established a finite element model of the contact surface of tubular members under loading, which has an important influence on the global static and dynamic responses of offshore structures. To sum up, the theoretical model of contact stiffness during unloading after the first loading of joint surface is very rare.

Because of this, based on the finite element contact model of Kogut and Etsion [14], a loading model of a single asperity was derived in this paper, and an unloading model of a single asperity was further derived by combining with the elastic-plastic sphere contact model of Etsion et al. [5]. On this basis, the contact stiffness model during the first loading and unloading of a fixed joint surface was established using the probability and statistics theory, and the influence law of the separation of joint surface on contact stiffness during loading and unloading was studied through model simulation.

\section{Model of Normal Contact Stiffness of a Single Asperity}

Figure 1 presents the deformation of a single asperity before and after contact with a rigid plane, where the dashed lines show the situation before deformation. The displacement of the rigid plane $\omega$ is the deformation of the asperity under the applied of the normal load $p . R$ is the curvature radius of the asperity summit.

2.1. Model of Normal Contact Stiffness of a Single Asperity during Loading. The critical deformation of a single asperity $\omega_{c}$, when it transforms from the elastic to the elastic-plastic deformation regime, is given by [5]

$$
\omega_{c}=\left(\frac{\pi K H}{2 E}\right)^{2} R
$$

where $H$ is the hardness of the softer material, which is related to its yield strength by $H=2.8 \sigma_{s}$ [15], $K$ is the hardness coefficient in the form $K=0.454+0.41 \nu$, where $v$ is Poisson's ratio of the softer material, And $E$, the comprehensive elastic modulus, is given in $1 / E=\left(1-v_{1}^{2}\right) / E_{1}+\left(1-v_{1}^{2}\right) / E_{2}$, where $E_{1}$ and $E_{2}$ and $v_{1}$ and $v_{2}$ are the elastic modulus and Poisson's ratios of the two materials, respectively. In this model, the rigid plane is smooth by $E_{2} \longrightarrow \infty$, so the comprehensive elastic modulus can be simplified as $E=E_{1} /\left(1-v_{1}^{2}\right)$.

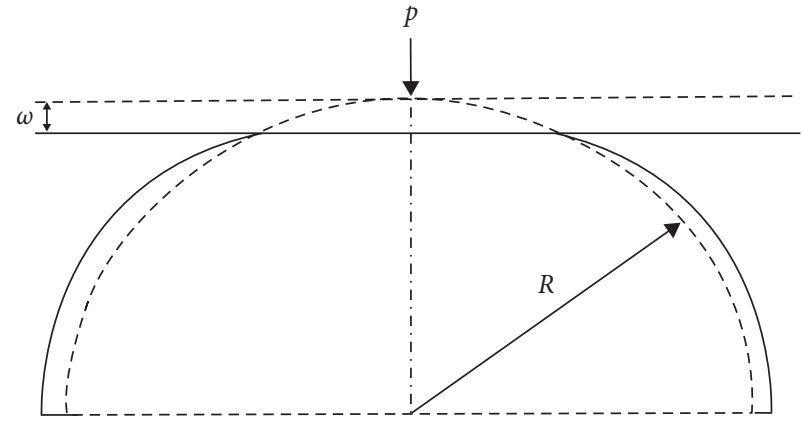

FIGURE 1: Contact deformation diagram of a single asperity with a rigid plane.

When $\omega \leq \omega_{c}$, a single asperity deforms elastically. According to Hertz theory [16], the normal contact load during loading can be expressed as follows:

$$
p_{\mathrm{el}}=\frac{4}{3} \mathrm{ER}^{1 / 2} \omega^{3 / 2}
$$

Therefore, the critical contact load of a single asperity $p_{c}$ that marks the transition from the elastic to the elasticplastic deformation regime can be expressed as follows:

$$
p_{c}=\frac{4}{3} \mathrm{ER}^{1 / 2} \omega_{c}^{3 / 2} .
$$

According to equation (2), the normal contact stiffness of a single asperity during loading $k_{\mathrm{el}}$ is given by

$$
k_{\mathrm{el}}=\frac{\mathrm{d} p_{\mathrm{el}}}{\mathrm{d} \omega}=2 \mathrm{ER}^{1 / 2} \omega^{1 / 2} .
$$

When $\omega_{c} \leq \omega \leq 110 \omega_{c}$, elastic-plastic deformation of a single asperity occurs. The normal contact load during loading can be expressed as follows:

$$
\begin{aligned}
& p_{\mathrm{epl} 1}=\frac{4.12}{3} \operatorname{ER}^{1 / 2} \omega_{c}^{3 / 2}\left(\frac{\omega}{\omega_{c}}\right)^{1.425}, \quad \omega_{c} \leq \omega \leq 6 \omega_{c}, \\
& p_{\mathrm{ep} 12}=\frac{5.6}{3} \operatorname{ER}^{1 / 2} \omega_{c}^{3 / 2}\left(\frac{\omega}{\omega_{c}}\right)^{1.263}, \quad 6 \omega_{c} \leq \omega \leq 110 \omega_{c} .
\end{aligned}
$$

Similarly, the normal contact stiffness of a single asperity during this loading is obtained as follows:

$$
\begin{gathered}
k_{\mathrm{epl} 1}=1.957 \mathrm{ER}^{1 / 2} \omega_{c}^{1 / 2}\left(\frac{\omega}{\omega_{c}}\right)^{0.425}, \quad \omega_{c} \leq \omega \leq 6 \omega_{c}, \\
k_{\mathrm{epl} 2}=2.3576 \mathrm{ER}^{1 / 2} \omega_{c}^{1 / 2}\left(\frac{\omega}{\omega_{c}}\right)^{0.263}, \quad 6 \omega_{c} \leq \omega \leq 110 \omega_{c} .
\end{gathered}
$$

When $\omega \geq 110 \omega_{c}$, a single asperity has a completely plastic deformation, in which there is no stiffness.

2.2. Model of Normal Contact Stiffness of a Single Asperity during Unloading. According to [5], the parameter that greatly influences the unloading of the asperity after the first loading is the maximum deformation during loading $\omega_{\max }$. 
When $\omega_{\max } \leq \omega_{c}$, only elastic deformation occurs during unloading after the first loading of the asperity. When $\omega_{\max }>\omega_{c}$, only elastic-plastic or full plastic deformation occurs during the first loading of an asperity, while partial elastic recovery deformation or no recovery deformation occurs during unloading after the first loading of the asperity.

When $\omega_{\max } \leq \omega_{c}$, the normal contact load $p_{\mathrm{eu}}$ and the normal contact stiffness $k_{\text {eu }}$ of the asperity during unloading can be expressed as follows:

$$
\begin{aligned}
& p_{\text {eu }}=\frac{4}{3} \mathrm{ER}^{1 / 2} \omega^{3 / 2}, \\
& k_{\text {eu }}=2 \mathrm{ER}^{1 / 2} \omega^{1 / 2} .
\end{aligned}
$$

When $\omega_{\max }>\omega_{c}$, the deformation of a single asperity before and after contacting with a rigid plane is shown in Figure 2, where the thin solid line represents the original topography of the asperity before loading and the thick solid line represents the topography of the asperity after fully unloading. When the loading load reaches the maximum value $P_{\max }$, the deformation of the asperity also reaches the maximum value $\omega_{\max }$, and the corresponding distance between the asperity and the rigid plane also reaches the minimum value $d_{\min }$. During unloading, due to the existence of some residual deformation, namely, the residual deformation $\omega_{\text {res }}$ and residual height of the unloading asperity $z_{\text {res }}$, its topography is different from that before loading. The parameters to characterize the spherical contour point of the unloaded asperity include residual deformation $\omega_{\text {res }}$ and residual nonuniform radius of curvature $R_{\text {res }}$.

According to [6], the relationship between normal contact load $p_{\text {epu }}$ and unloading deformation of asperity $\omega$ is

$$
p_{\text {epu }}=p_{\max }\left(\frac{\omega-\omega_{\text {res }}}{\omega_{\max }-\omega_{\text {res }}}\right)^{n_{p}},
$$

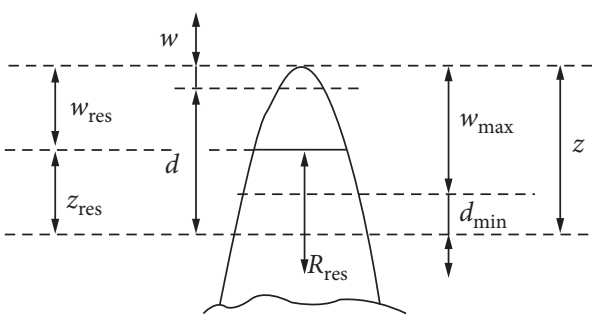

FIgURE 2: Schematic diagram of a deformed asperity before and after loading and unloading.

where the index of the plastic stage is given by $n_{p}=1.5\left(\omega_{\max } / \omega_{c}\right)^{-0.0331}$.

The residual deformation $\omega_{\text {res }}$ and the radius of residual nonuniform curvature $R_{\text {res }}$ depend on the maximum load at the beginning of the unloading $p_{\max }$. According to relations (5) and (6) between contact load and deformation, the maximum load $p_{\max }$ can be deduced as follows:

$$
\begin{gathered}
p_{\max 1}=\frac{4.12}{3} \operatorname{ER}^{1 / 2} \omega_{c}^{3 / 2}\left(\frac{\omega_{\max }}{\omega_{c}}\right)^{1.425}, \quad \omega_{c} \leq \omega_{\max } \leq 6 \omega_{c}, \\
p_{\max 2}=\frac{5.6}{3} \mathrm{ER}^{1 / 2} \omega_{c}^{3 / 2}\left(\frac{\omega_{\max }}{\omega_{c}}\right)^{1.263}, \quad 6 \omega_{c} \leq \omega_{\max } \leq 110 \omega_{c} .
\end{gathered}
$$

By substituting equations (12), (13) into equation [11], respectively, and making further differentiation, the unloading stiffness of an elastic-plastic deformable asperity can be deduced as follows:

$$
\begin{gathered}
k_{\text {epu } 1}=\frac{4.12 \mathrm{ER}^{1 / 2} \omega_{c}^{3 / 2} n_{p}}{3\left(\omega_{\max }-\omega_{\text {res }}\right)}\left(\frac{\omega_{\max }}{\omega_{c}}-1\right)^{1.425}\left(\frac{\omega-\omega_{\text {res }}}{\omega_{\text {max }}-\omega_{\text {res }}}\right)^{n_{p}-1}, \quad \omega_{c} \leq \omega_{\max } \leq 6 \omega_{c}, \\
k_{\text {epu2 }}=\frac{5.6 \mathrm{ER}^{1 / 2} \omega_{c}^{3 / 2} n_{p}}{3\left(\omega_{\max }-\omega_{\text {res }}\right)}\left(\frac{\omega_{\max }}{\omega_{c}}-1\right)^{1.263}\left(\frac{\omega-\omega_{\text {res }}}{\omega_{\max }-\omega_{\text {res }}}\right)^{n_{p}-1}, \quad 6 \omega_{c} \leq \omega_{\max } \leq 110 \omega_{c} .
\end{gathered}
$$

In the same way, the plastically deformable asperity does not recover. There is no contact stiffness during unloading.

In [5], the ratio relation $\omega_{\text {res }} / \omega_{\max }$ was given as follows:

$$
\frac{\omega_{\text {res }}}{\omega_{\max }}=\left(1-\frac{1}{\left(\omega_{\max } / \omega_{c}\right)^{0.28}}\right)\left(1-\frac{1}{\left(\omega_{\max } / \omega_{c}\right)^{0.69}}\right) \text {. }
$$

\section{Statistical Model of Normal Contact Stiffness of Joint Surface}

3.1. Statistical Model of Normal Contact Stiffness of Joint Surface during Loading. Based on Greenwood and Williamson's model (GW model), this paper assumes that there is no interaction between asperities, and all deformation is 
limited to the contacting asperities. So, the fixed joint surface is equivalent to the contact between a rough surface and a smooth rigid plane, as shown in Figure 3. $Z$ is the height of asperities, $d$ is the distance between the mean of asperity heights and the rigid plane, $h$ is the distance between the mean of surface heights and the rigid plane, and $y_{s}$ is the distance between the mean of asperity heights and the mean of surface heights, satisfying the relation $d=h-y_{s}$. The rough surface is isotropic, and its morphology is defined by three independent parameters: the area density of asperities $\eta$, the ratio of the standard deviation of asperity heights to the standard deviation of surface heights $\sigma_{s} / \sigma$, and the radius of curvature of asperity summit $R$.

The relation of the ratio $\sigma_{s} / \sigma$ can be expressed as

$$
\frac{\sigma_{s}}{\sigma}=\sqrt{1-\frac{3.717 \times 10^{-4}}{\beta^{2}}}
$$

where $\beta$ is a dimensionless surface roughness parameter in the form:

$$
\beta=\eta R \sigma
$$

Assuming that there are $N$ asperities on the nominal contact area $A_{n}$, the expected number of contact asperities on the joint surface $n$ is given by

$$
n=N \int_{d}^{\infty} \phi(z) d_{z}=\eta A_{n} \int_{d}^{\infty} \phi(z) d_{z}
$$

where $\phi(z)$ is the probability density function of the normal distribution of asperity heights.

The distribution function $\phi^{*}\left(z^{*}\right)$ of dimensionless asperity heights $z^{*}$ is described by a dimensionless Gaussian standard probability density function in the form:

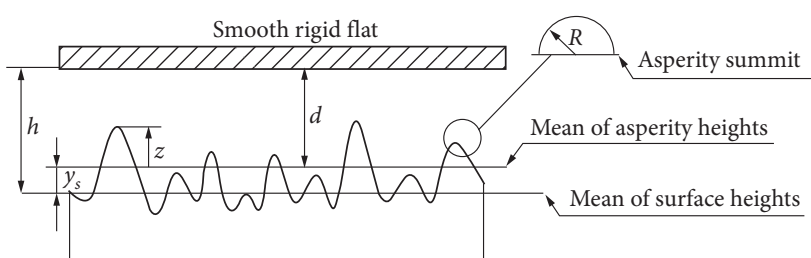

Figure 3: Schematic diagram of contact between a rough surface and a smooth rigid surface.

$$
\phi^{*}\left(z^{*}\right)=\frac{1}{\sqrt{2 \pi}} \frac{\sigma}{\sigma_{s}} \exp \left(-\frac{1}{2}\left(\frac{\sigma}{\sigma_{s}}\right)^{2}\left(z^{*}\right)^{2}\right) .
$$

The dimensionless distance $y_{s}^{*}$ between the mean of asperity heights and the mean of surface heights is given by [6]

$$
y_{s}^{*}=h^{*}-d^{*}=\frac{1.5}{\sqrt{108 \pi \beta}}
$$

The random dimensionless interference of a single asperity can be expressed as follows:

$$
\omega^{*}=z^{*}-d^{*} \text {. }
$$

In this paper, the plastic index form proposed by GW is adopted:

$$
\psi=\left(\omega_{c}^{*} \frac{\sigma}{\sigma_{s}}\right)^{-1 / 2} .
$$

According to equation (1), the normal contact stiffness of the joint surface during loading is

$$
\begin{aligned}
K_{l}= & \eta A_{n} \int_{d}^{d+\omega_{c}} k_{\mathrm{el}} \phi(z) \mathrm{d} z+\eta A_{n} \int_{d+\omega_{c}}^{d+6 \omega_{c}} k_{\mathrm{epl} 1} \phi(z) \mathrm{d} z+\eta A_{n} \int_{d+6 \omega_{c}}^{d+110 \omega_{c}} k_{\mathrm{epl} 2} \phi(z) \mathrm{d} z \\
= & 2 \mathrm{ER}^{1 / 2} \eta A_{n} \int_{d}^{d+\omega_{c}} \omega^{1 / 2} \phi(z) \mathrm{d} z+0.9785 \pi K H R \eta A_{n} \int_{d+\omega_{c}}^{d+6 \omega_{c}}\left(\frac{\omega}{\omega_{c}}\right)^{0.425} \phi(z) \mathrm{d} z \\
& +1.1788 \pi K H R \eta A_{n} \int_{d+6 \omega_{c}}^{d+110 \omega_{c}}\left(\frac{\omega}{\omega_{c}}\right)^{0.263} \phi(z) \mathrm{d} z .
\end{aligned}
$$


The dimensionless form of equation (24) is

$$
\begin{aligned}
K_{l}^{*}= & \frac{K_{l}}{\left(\mathrm{EA}_{n}\right) / \sigma}=2 \beta\left(\frac{\sigma}{R}\right)^{1 / 2} \int_{h^{*}-y_{s}^{*}}^{h^{*}-y_{s}^{*}+\omega_{c}^{*}}\left(\omega^{*}\right)^{1 / 2} \phi^{*}\left(z^{*}\right) \mathrm{d} z^{*} \\
& +\frac{0.9785 \pi K H \beta}{E} \int_{h^{*}-y_{s}^{*}+\omega_{c}^{*}}^{h^{*}-y_{s}^{*}+6 \omega_{c}^{*}}\left(\frac{\omega^{*}}{\omega_{c}^{*}}\right)^{0.425} \phi^{*}\left(z^{*}\right) \mathrm{d} z^{*} \\
& +\frac{1.1788 \pi K H \beta}{E} \int_{h^{*}-y_{s}^{*}+6 \omega_{c}^{*}}^{h^{*}-y_{s}^{*}+110 \omega_{c}^{*}}\left(\frac{\omega^{*}}{\omega_{c}^{*}}\right)^{0.263} \phi^{*}\left(z^{*}\right) \mathrm{d} z^{*} .
\end{aligned}
$$

3.2. Statistical Model of Normal Contact Stiffness of Fixed Joint Surface during Unloading after the First Loading. The normal contact stiffness of joint surface during unloading can be expressed as

$$
\begin{aligned}
K_{u}= & 2 \mathrm{ER}^{1 / 2} \eta A_{n} \int_{d_{\min }}^{d_{\min }+\omega_{c}} \omega^{1 / 2} \phi(z) \mathrm{d} z \\
& +\frac{1.03(\pi K H)^{3} R^{2} \eta A_{n}}{6 E^{2}} \int_{d_{\min }+\omega_{c}}^{d_{\min }+6 \omega_{c}} \frac{n_{p}}{\omega_{\max }-\omega_{\text {res }}}\left(\frac{\omega_{\max }}{\omega_{c}}\right)^{1.425}\left(\frac{\omega-\omega_{\text {res }}}{\omega_{\max }-\omega_{\text {res }}}\right)^{n_{p}-1} \phi(z) \mathrm{d} z \\
& +\frac{0.7(\pi K H)^{3} R^{2} \eta A_{n}}{3 E^{2}} \int_{d_{\min }+6 \omega_{c}}^{d_{\min }+110 \omega_{c}} \frac{n_{p}}{\omega_{\max }-\omega_{\text {res }}}\left(\frac{\omega_{\max }}{\omega_{c}}\right)^{1.263}\left(\frac{\omega-\omega_{\text {res }}}{\omega_{\max }-\omega_{\text {res }}}\right)^{n_{p}-1} \phi(z) \mathrm{d} z .
\end{aligned}
$$

The dimensionless form of the above equation is

$$
\begin{aligned}
K_{u}^{*}= & \frac{K_{u}}{\left(\mathrm{EA}_{n}\right) / \sigma}=2 \beta\left(\frac{\sigma}{R}\right)^{1 / 2} \int_{h_{\min }^{*}-y_{s}^{*}}^{h_{\min }^{*}-y_{s}^{*}+\omega_{c}^{*}}\left(\omega^{*}\right)^{1 / 2} \phi^{*}\left(z^{*}\right) d z^{*} \\
& +\frac{1.03(\pi K H)^{3} R \beta}{6 E^{3}} \int_{h_{\min }^{*}-y_{s}^{*}+\omega_{c}^{*}}^{h_{\min }^{*}-y_{s}^{*}+6 \omega_{c}^{*}} \frac{n_{p}^{*}}{\omega_{\max }^{*}-\omega_{\mathrm{res}}^{*}}\left(\frac{\omega_{\max }^{*}}{\omega_{c}^{*}}\right)^{1.425}\left(\frac{\omega^{*}-\omega_{\mathrm{res}}^{*}}{\omega_{\max }^{*}-\omega_{\mathrm{res}}^{*}}\right)^{n_{p}^{*}-1} \phi^{*}\left(z^{*}\right) d z^{*} \\
& +\frac{0.7(\pi K H)^{3} R \beta}{3 E^{3}} \int_{h_{\min }^{*}-y_{s}^{*}+6 \omega_{c}^{*}}^{h_{\min }^{*}-y_{s}^{*}+110 \omega_{c}^{*}} \frac{n_{p}^{*}}{\omega_{\max }^{*}-\omega_{\mathrm{res}}^{*}}\left(\frac{\omega_{\max }^{*}}{\omega_{c}^{*}}\right)^{1.263}\left(\frac{\omega^{*}-\omega_{\mathrm{res}}^{*}}{\omega_{\max }^{*}-\omega_{\mathrm{res}}^{*}}\right)^{n_{p}^{*}-1} \phi^{*}\left(z^{*}\right) d z^{*},
\end{aligned}
$$

where $n_{p}^{*}=1.5\left(\omega_{\max }^{*} / \omega_{c}^{*}\right)^{-0.0331}$.

\section{Simulation and Result Analysis of the Model}

It can be seen from equation (25) that the dimensionless loading normal contact stiffness $K_{l}^{*}$ is a function of the standard deviation of surface heights $\sigma$, the radius of curvature at the initial summit of asperity $R$, the dimensionless surface roughness parameter $\beta$, the dimensionless surface mean separation $h^{*}$, and so on. It can be seen from equation (27) that the dimensionless unloading normal contact stiffness $K_{u}^{*}$ is a function of the standard deviation of surface heights $\sigma$, the radius of curvature at the initial summit of asperity $R$, the dimensionless surface roughness parameter $\beta$, the dimensionless surface mean separation $h^{*}$, the dimensionless residual deformation $\omega_{\text {res }}^{*}$, and so on. And, it is not affected by residual nonuniform curvature radius $R_{\text {res. }}$. In the simulation analysis, the parameters are given such as the elastic modulus $E_{1}=E_{2}=2.07 \times 10^{11} \mathrm{~Pa}$, Poisson's ratio $v_{1}=v_{2}=0.29$, the hardness $H=1.96 \times 10^{9} \mathrm{~Pa}$, the radius of curvature at the initial summit of asperity $R=6.89 \times 10^{-4} \mathrm{~mm}$, and the dimensionless surface roughness parameters $\beta$ and $\sigma / R$ [17] (shown in Table 1). Equations (25) and (27) are simulated by using the data of each variable, and the corresponding results are shown in Figure 4.

It can be seen from Figure 4 that the normal contact stiffness of joint surface during loading and unloading is a nonlinear function of the mean surface separation and decreases with the increase of the mean surface separation. When the plastic index is smaller, the contact between asperities is more elastic, so the normal contact stiffness curves of joint surface during loading and unloading are close. When the plastic index is larger, the plastic deformation cannot recover due to the large proportion of the plastically deformed asperities, so the normal contact stiffness decreases rapidly during unloading. 
TABLE 1: Dimensionless surface roughness parameters [17].

\begin{tabular}{lcr}
\hline Number & $\beta$ & $\sigma / R$ \\
\hline 1 & 0.0339 & $1.600 \times 10^{-4}$ \\
2 & 0.0476 & $6.576 \times 10^{-4}$ \\
3 & 0.0541 & $1.144 \times 10^{-3}$ \\
4 & 0.0601 & $1.770 \times 10^{-3}$ \\
\hline
\end{tabular}

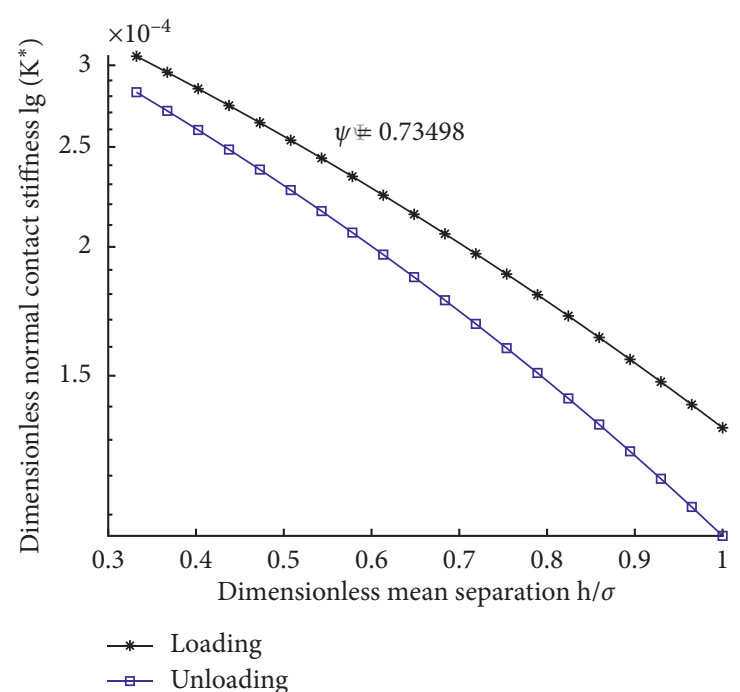

(a)

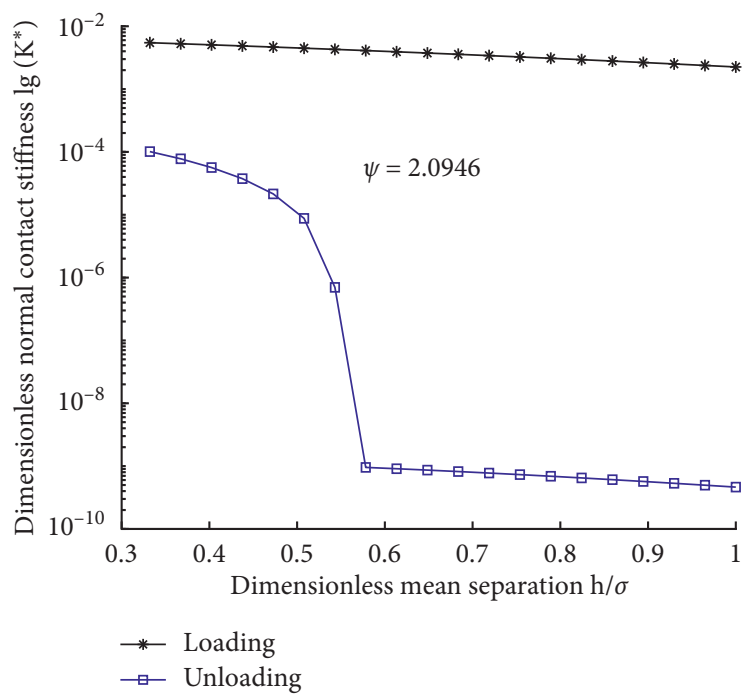

(c)

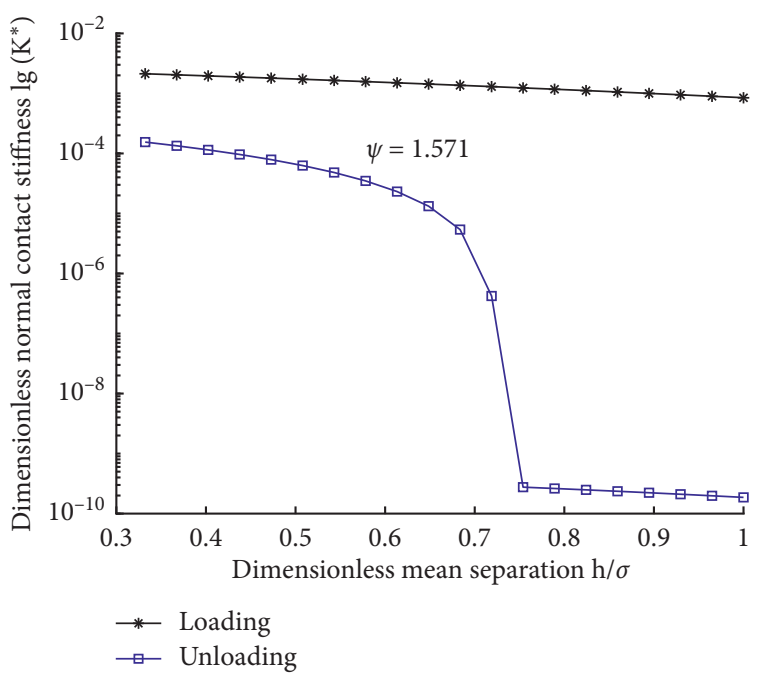

(b)

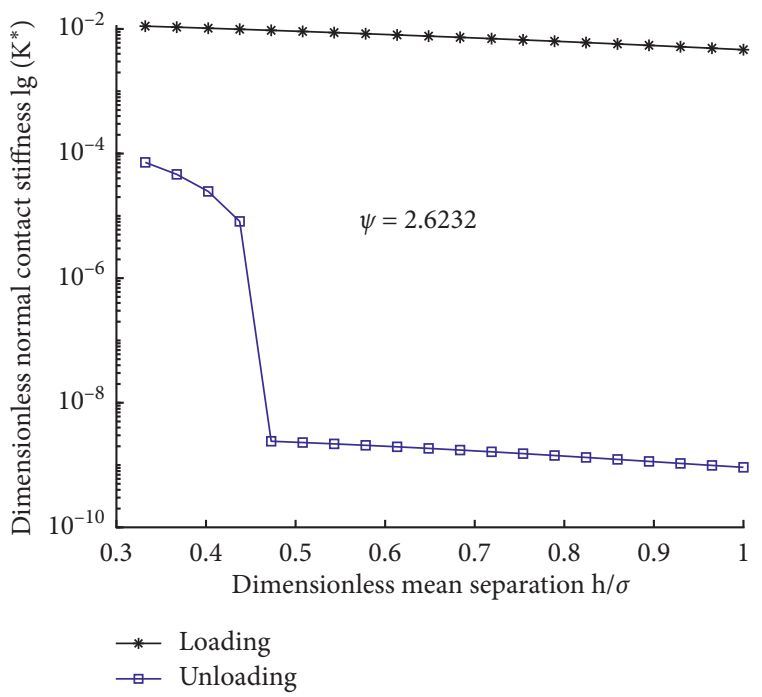

(d)

Figure 4: Influence of $h^{*}$ on $\lg \left(K^{*}\right)$. (a) $\psi=0.73498$. (b) $\psi=1.571$. (c) $\psi=2.0946$. (d) $\psi=2.6232$.

In order to verify the effectiveness of the normal unloading contact stiffness model of joint surface established in this paper, a comparative analysis is made between the model in this paper and the model in [10] (shown in Figure 5). It can be seen from Figure 5 that the unloading stiffness in both contact models decreases with increase of mean surface separation, but there is a large deviation between them. According to the definition of plastic index $\psi$ in the GW model, when $\psi<0.6$, elastic deformation of asperities occurs even if the load is very large; when $0.6 \leq \psi \leq 1$, the deformation of asperities depends on magnitude of load, and the larger the value $\psi$ is, the larger the proportion of plastic deformation is. Therefore, when $\psi \leq 1$, the normal contact stiffness of fixed joint surface is larger during unloading. When $\psi>1$, even if the load is very small, the deformation of asperities is plastic, while the plastic deformation cannot 


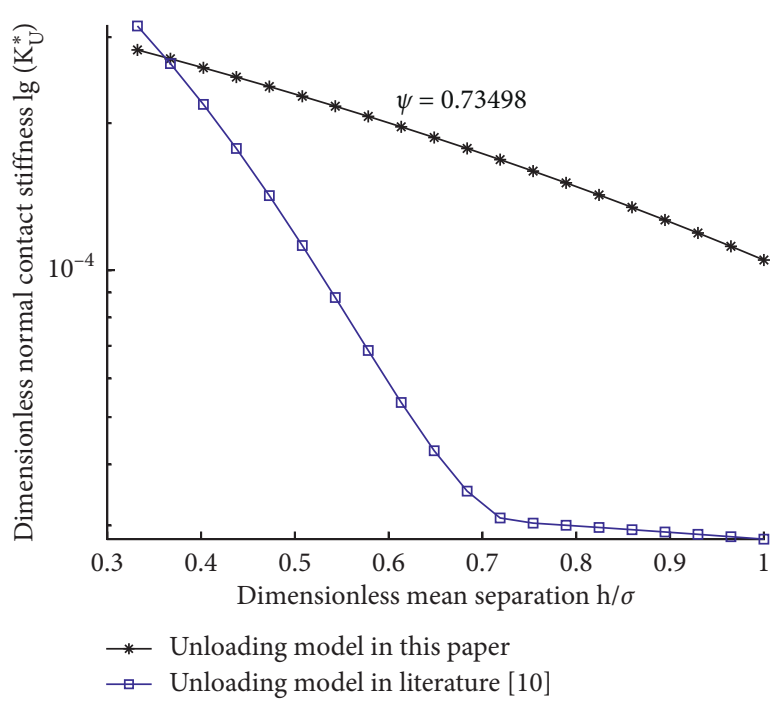

(a)

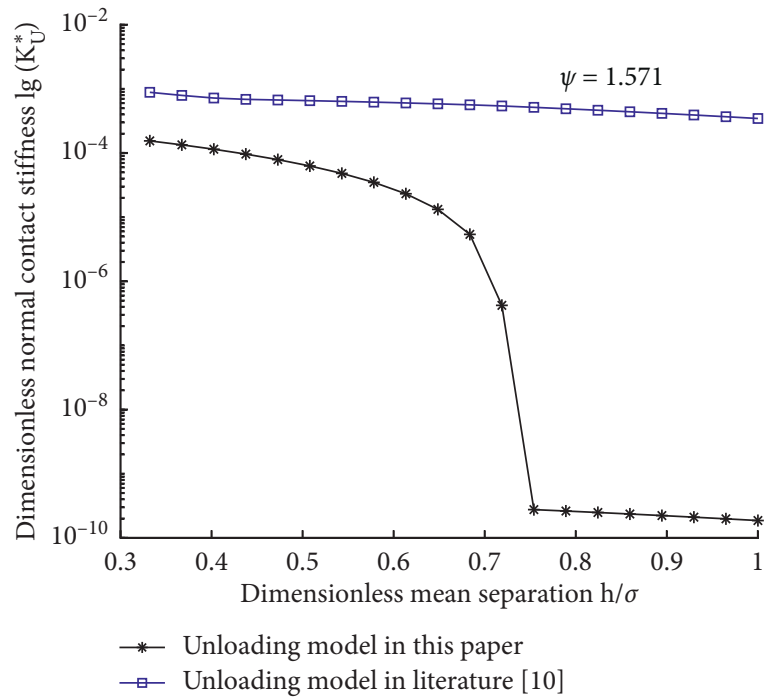

(b)

Figure 5: A comparison between the unloading model in this paper and that in [10]. (a) $\psi=0.73498$. (b) $\psi=1.571$.

recover, so the normal contact stiffness of fixed joint surface is smaller during unloading. Therefore, the normal unloading contact stiffness model of joint surface established in this paper will be more in line with the actual situation.

\section{Conclusions}

In this paper, a statistical model of the normal contact stiffness of fixed joint surface during unloading after the first load is established, and a simulation analysis is carried out on the model to study the influence of the mean surface separation on the normal contact stiffness. The findings are as follows:

(1) Dimensionless loading and unloading normal contact stiffness decreases with the increase of the dimensionless mean surface separation for different plastic indexes.

(2) The smaller the plastic index is, the less obvious the difference between loading and unloading caused by plastic deformation would be. Therefore, the normal contact stiffness curve during unloading is close to that during loading. On the contrary, with the increase of plastic index, the normal contact stiffness during unloading is obviously less than that during loading.

(3) However, the larger the plastic index is, the slower the loading normal contact stiffness decreases, while the faster the unloading normal contact stiffness decreases.

Unfortunately, our paper did have some limitations and shortcomings, which will be verified by supplementary experiments in the future.

\section{Data Availability}

The data used to support the findings of this study are available from the corresponding author upon request.

\section{Conflicts of Interest}

The authors declare that there are no conflicts of interest regarding the publication of this paper.

\section{Acknowledgments}

This work was supported by Shanxi Provincial Natural Science Foundation of China (Grant no. 201901D111248), Shanxi Provincial "1331" Engineering Key Discipline Construction Project of China, and Shanxi Provincial Graduate Education Innovation Project of China (Grant No. 2020BY112).

\section{References}

[1] Y. Ren and C. F. Beards, "Identification of "effective" linear joints using coupling and joint identification techniques," Journal of Vibration and Acoustics, vol. 120, no. 2, pp. 331-338, 1998.

[2] Y.-Y. Lin and C. Y. Hui, "Mechanics of contact and adhesion between viscoelastic spheres: an analysis of hysteresis during loading and unloading," Journal of Polymer Science Part B: Polymer Physics, vol. 40, no. 9, pp. 772-793, 2002.

[3] S. L. Yan and L. Y. Li, "Finite element analysis of cyclic indentation of an elastic-perfectly plastic half-space by a rigid sphere," Proceedings of the Institution of Mechanical Engineers, Part C: Journal of Mechanical Engineering Science, vol. 217, no. 5, pp. 505-514, 2003.

[4] R. E. Jones, "Models for contact loading and unloading of a rough surface," International Journal of Engineering Science, vol. 42, no. 17-18, pp. 1931-1947, 2004.

[5] I. Etsion, Y. Kligerman, and Y. Kadin, "Unloading of an elastic-plastic loaded spherical contact," International Journal of Solids and Structures, vol. 42, no. 13, pp. 3716-3729, 2005.

[6] Y. Kadin, Y. Kligerman, and I. Etsion, "Unloading an elasticplastic contact of rough surfaces," Journal of the Mechanics and Physics of Solids, vol. 54, no. 12, pp. 2652-2674, 2006. 
[7] Y. Zait, Y. Kligerman, and I. Etsion, "Unloading of an elasticplastic spherical contact under stick contact condition," International Journal of Solids and Structures, vol. 47, no. 7-8, pp. 990-997, 2010.

[8] J. H. Zhao, S. Nagao, and Z. L. Zhang, "Loading and unloading of a spherical contact: from elastic to elastic-perfectly plastic materials," International Journal of Mechanical Sciences, vol. 56, no. 1, pp. 70-76, 2012.

[9] W. Fu, L. T. Lou, and Z. Q. Gao, "Theoretical model for the contact stiffness and damping of mechanical joint surface," Journal of Mechanical Engineering, vol. 53, no. 9, pp. 073-082, 2017.

[10] R. An, X. L. Zhang, and Y. S. Wang, "Statistical model of unloading normal contact stiffness of joint surface," Journal of Taiyuan University of Science and Technology, vol. 38, no. 5, pp. 373-377, 2017.

[11] H. Nassiraei, "Local joint flexibility of CHS X-joints reinforced with collar plates in jacket structures subjected to axial load," Applied Ocean Research, vol. 93, pp. 1-10, 2019.

[12] H. Nassiraei, "Local joint flexibility of CHS T/Y-connections strengthened with collar plate under in-plane bending load: parametric study of geometrical effects and design formulation," Ocean Engineering, vol. 202, pp. 1-11, 2020.

[13] H. Nassiraei, "Geometrical effects on the LJF of tubular T/Yjoints with doubler plate in offshore wind turbines," Ships and Offshore Structures, vol. 9, pp. 1-11, 2020.

[14] L. Kogut and I. Etsion, "Elastic-plastic contact analysis of a sphere and a rigid flat," Journal of Applied Mechanics, vol. 69, no. 5, pp. 657-662, 2002.

[15] D. Tabor, The Hardness of Metals, Clarendon Press, Oxford, UK, 1951.

[16] K. L. Johnson, Contact Mechanics, Cambridge University Press, Cambridge, UK, 1985.

[17] L. Li, A. J. Cai, and L. G. Cai, "Micro-contact model of boltedjoint interface," Journal of Mechanical Engineering, vol. 52, no. 7, pp. 205-212, 2016. 